From all studies so far we are still left with the fundamental question: Do non-SMCs from the adventitia, host vessel, or bone marrow transdifferentiate to become bona fide SMCs? It is possible that these cells are expressing only a few SMC differentiation markers and/or still expressing non-SMC genes. Finally, and most importantly, we ask, are the results in these animal models relevant to the development of atherosclerosis in humans? Can these same cells be identified in humans by Sca-1 antigen? The novel and exciting studies by Hu et al. in this issue of the JCI implicate potential contributions by a putative adventitial progenitor cell population in TA and might open yet another chapter in the long quest to define origins of cells in intimal lesions and their mechanistic contributions to the pathogenesis of atherosclerosis.

Mark H. Hoofnagle and Brian R. Wamhoff contributed equally to this work.

Address correspondence to: Gary K. Owens, Department of Molecular Physiology and Biological Physics, University of Virginia, MR5 Room 1220, 415 Lane Road, PO Box 801394, Charlottesville, Virginia 22908,
USA. Phone: (434) 924-2652; Fax: (434) 982-0055; E-mail: gko@virginia.edu.

1. Ross, R., and Glomset, J.A. 1973. Atherosclerosis and the arterial smooth muscle cell: proliferation of smooth muscle is a key event in the genesis of the lesions of atherosclerosis. Science. 180:1332-1339.

2. Sata, M., et al. 2002. Hematopoietic stem cells differentiate into vascular cells that participate in the pathogenesis of atherosclerosis. Nat. Med. 8:403-409.

3. Shimizu, K., et al. 2001. Host bone-marrow cells are a source of donor intimal smooth-muscle-like cells in murine aortic transplant arteriopathy. Nat. Med. 7:738-741.

4. Glaser, R., Lu, M.M., Narula, N., and Epstein, J.A. 2002. Smooth muscle cells, but not myocytes, of host origin in transplanted human hearts. Circula tion. 106:17-19.

5. Simper, D., Stalboerger, P.G., Panetta, C.J., Wang, S., and Caplice, N.M. 2002. Smooth muscle progenitor cells in human blood. Circulation. 106:1199-1204.

6. Owens, G.K., Kumar, M.S., and Wamhoff, B.R. 2004. Molecular regulation of vascular smooth muscle cell differentiation in development and disease. Physiol. Rev. In press.

7. Campbell, J.H., Han, C.L., and Campbell, G.R. 2001. Neointimal formation by circulating bone marrow cells. Ann. N. Y. Acad. Sci. 947:18-24.

8. Hu, Y., et al. 2002. Both donor and recipient origins of smooth muscle cells in vein graft atherosclerotic lesions. Circ. Res. 91:13e-20e.

9. $\mathrm{Hu}, \mathrm{Y}$., et al. 2002. Smooth muscle cells in transplant atherosclerotic lesions are originated from recipients, but not bone marrow progenitor cells. Circulation. 106:1834-1839.

10. Hu, Y., et al. 2004. Abundant progenitor cells in the adventitia contribute to atherosclerosis of vein grafts in ApoE-deficient mice. J. Clin. Invest. 113:1258-1265. doi:10.1172/JCI200419628.

11. Bjornson, C.R., Rietze, R.L., Reynolds, B.A., Magli, M.C., and Vescovi, A.L. 1999. Turning brain into blood: a hematopoietic fate adopted by adult neural stem cells in vivo. Science. 283:534-537.

12. Lagasse, E., et al. 2000. Purified hematopoietic stem cells can differentiate into hepatocytes in vivo. Nat. Med. 6:1229-1234.

13. Wang, X., et al. 2003. Cell fusion is the principal source of bone-marrow-derived hepatocytes. Nature. 422:897-901.

14. Wagers, A.J., Sherwood, R.I., Christensen, J.L., and Weissman, I.L. 2002. Little evidence for developmental plasticity of adult hematopoietic stem cells. Science. 297:2256-2259.

15. Terada, N., et al. 2002. Bone marrow cells adopt the phenotype of other cells by spontaneous cell fusion. Nature. 416:542-545

16. Ying, Q.L., Nichols, J., Evans, E.P., and Smith, A.G. 2002. Changing potency by spontaneous fusion. Nature. 416:545-548.

17. Ianus, A., Holz, G.G., Theise, N.D., and Hussain, M.A. 2003. In vivo derivation of glucose-competent pancreatic endocrine cells from bone marrow without evidence of cell fusion. J. Clin. Invest. 111:843-850. doi:10.1172/JCI200316502.

18. Spees, J.L., et al. 2003. Differentiation, cell fusion, and nuclear fusion during ex vivo repair of epithelium by human adult stem cells from bone marrow stroma. Proc. Natl. Acad. Sci. U. S. A. 100:2397-2402.

19. Saiura, A., et al. 2003. Little evidence for cell fusion between recipient and donor-derived cells. J. Surg. Res. 113:222-227.

20. Camargo, F.D., Finegold, M., and Goodell, M.A. 2004. Hematopoietic myelomonocytic cells are the major source of hepatocyte fusion partners. J. Clin. Invest. 113:1266-1270. doi:10.1172/JCI200421301.

\title{
Anemia of inflammation: the cytokine-hepcidin link
}

\author{
Nancy C. Andrews \\ Children's Hospital, Howard Hughes Medical Institute, Harvard Medical School, and Dana-Farber Cancer Institute, Boston, Massachusetts, USA.
}

\begin{abstract}
The anemia of inflammation, commonly observed in patients with chronic infections, malignancy, trauma, and inflammatory disorders, is a well-known clinical entity. Until recently, we understood little about its pathogenesis. It now appears that the inflammatory cytokine IL-6 induces production of hepcidin, an iron-regulatory hormone that may be responsible for most or all of the features of this disorder (see the related article beginning on page 1271).
\end{abstract}

In 1932, Locke et al. made the important observation that infection was associated with hypoferremia (low serum iron), providing a partial explanation for the common finding of anemia in patients with chronic infections (1). Cartwright and Wintrobe went on to show that the anemia associated with infection was indistinguishable from the anemia of inflam-

Conflict of interest: The author has declared that no conflict of interest exists.

Citation for this article: J. Clin. Invest. 113:1251-1253 (2004). doi:10.1172/JCI200421441. mation, and established that hypoferremia resulted from reticuloendothelial sequestration of iron and interruption of intestinal iron absorption $(2,3)$. Cartwright and Lee recognized that similar findings could be induced in mice by exposure to bacterial endotoxin (4). Others correlated the anemia of inflammation with elaboration of inflammatory cytokines, and ascribed changes in iron metabolism to the effects of these cytokines (5). Cytokines have been shown to modulate the expression of iron transport and storage proteins (6), but it was not clear that these changes accounted for the abnormalities of iron homeostasis observed in the anemia of inflammation.

\section{The roles of hepcidin and IL- 6}

Over the past two years, a variety of experiments have converged to establish a role for hepcidin, a liver-derived peptide regulator of iron homeostasis, as a key mediator of hypoferremia in inflammation (7-9). In an elegant report in this issue of the JCI, Nemeth, Rivera, and colleagues have elucidated an important link between inflammatory cytokines and hepcidin (10). Using both mice and humans as experimental models, they have shown that IL- 6 acts directly on hepatocytes to stimulate hepcidin production. Hepcidin, in turn, acts as a negative regulator of intestinal iron absorption and macrophage iron release.

In previous work Nemeth, Rivera, and colleagues showed that IL- 6 induced hepcidin expression in hepatic cells (9). 


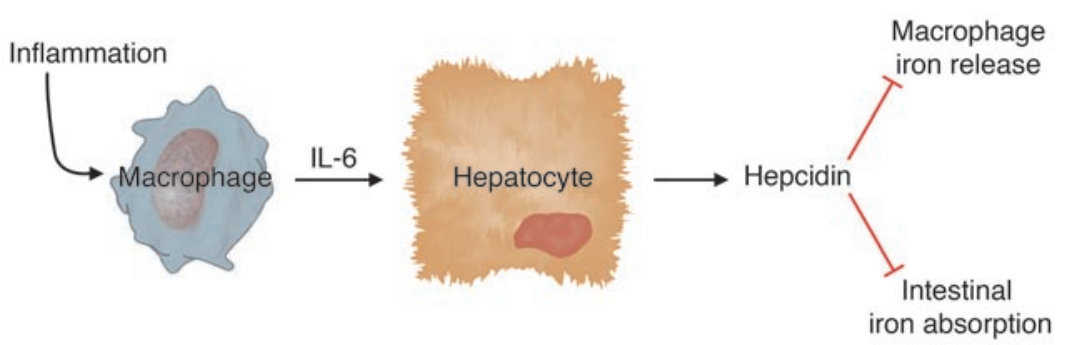

Here, they have replicated this effect using conditioned medium from endotoxintreated macrophages and shown that a neutralizing antibody against IL-6 blocked hepcidin induction (10). Other inflammatory cytokines did not stimulate hepcidin production; in fact, TNF- $\alpha$ inhibited it.

Cytokines have all sorts of effects on cultured cells, and it was important to show that IL-6 induction of hepcidin occurred in vivo and triggered hypoferremia, as predicted. First, Nemeth, Rivera, et al. used turpentine injection to cause inflammatory abscesses in wild-type and IL- 6 knockout mice and analyzed the responses (10). Wildtype mice had increased hepcidin expression and a substantial decrease in serum iron levels. In contrast, IL-6 knockout mice had no increase in hepcidin expression and no decrease in serum iron. A complementary experiment carried out in human volunteers showed that IL- 6 infusion stimulated urinary hepcidin excretion within 2 hours and induced hypoferremia. Taken together, these data provide strong support for the conclusions that IL- 6 is a primary inducer of hepcidin expression and that increased hepcidin expression results in hypoferremia (Figure 1). This is gratifyingly consistent with clinical observations that hypoferremia occurs very quickly after the onset of inflammation.

Earlier studies had shown that rodents with induced iron overload also had increased hepcidin expression $(11,12)$, presumably to try to compensate for iron excess. However, the signal to increase hepcidin expression was unknown. Here, Nemeth, Rivera, and colleagues have shown that IL-6 is not involved in the regulation of hepcidin in response to iron (10). Furthermore, their data suggest that hepcidin levels are not simply responding to increased iron stores. In human volunteers, urinary hepcidin levels were boosted soon after a single dose of oral iron, which should have no significant effect on iron stores. Perhaps the serum iron level, known to increase transiently after iron inges- tion, might itself be the signal to induce hepcidin expression. Alternatively, the signal might relate to the degree of iron saturation of serum transferrin.

However, if transferrin iron saturation modulates hepcidin expression, other signals can clearly override its effects. The IL-6-mediated inflammatory induction of hepcidin does not appear to be offset by the hypoferremia it causes, at least in the short term. And mice with thalassemia intermedia (which presumably have elevated serum iron) have decreased hepcidin expression (13), as occurs in other mouse models with increased erythroid iron demand $(7,8)$.

In my opinion, this report from Nemeth, Rivera, et al. (10) leaves little room for doubt about the importance of hepcidin in the pathogenesis of anemia of inflammation. This was challenged by a recent report that concluded that elevated serum hepcidin levels were not useful in the diagnosis of the anemia of inflammation (14). However, that study did not provide data to support the authors' contention that they had developed a sensitive, specific test for serum hepcidin. Furthermore, as they also pointed out, it was not clear that serum measurements were as useful as urinary hepcidin measurements. Hepcidin gene expression seems to be exquisitely sensitive to regulation, and the circulating peptide is small enough to be quantitatively filtered by the kidneys. Urine samples probably provide a better indication of recent hepcidin expression than individual serum samples.

\section{A possible treatment?}

If inflammatory induction of hepcidin causes hypoferremia, it is logical to predict that inhibition of hepcidin expression or activity would ameliorate the anemia of inflammation. Would that be advantageous? Perhaps, particularly in noninfectious inflammatory disorders. We know that patients (15) and mice (16) lacking hepcidin have increased intestinal iron absorption and increased serum iron, but

\section{Figure 1}

Regulation of hepcidin production in inflammation. Inflammation leads to macrophage elaboration of IL-6, which acts on hepatocytes to induce hepcidin production. Hepcidin inhibits macrophage iron release and intestinal iron absorption, leading to hypoferremia.

this is unlikely to be harmful in the short term. However, there may be more cause for concern in patients with infections or malignancy. Decreased serum iron is believed to contribute to host defense against invading pathogens and tumor cells (17), and hepcidin itself has antimicrobial properties of uncertain importance (18). If hepcidin antagonists become available, careful clinical trials will be required to define appropriate indications for their use.

Address correspondence to: Nancy C. Andrews, Children's Hospital, Howard Hughes Medical Institute, and Harvard Medical School, 300 Longwood Avenue, Boston, Massachusetts 02115-5737, USA. Phone: (617) 919-2116; Fax: (617) 730-0934; E-mail: nandrews@enders.tch.harvard.edu.

1. Locke, A., Main, E.R., and Rosbach, D.O. 1932. The copper and non-hemoglobinous iron contents of the blood serum in disease. J. Clin. Invest. 11:527-542.

2. Cartwright, G.E., and Wintrobe, M.M. 1952. The anemia of infection. XVII. A review. Adv. Intern. Med. 5:165-226.

3. Cartwright, G.E. 1966. The anemia of chronic disorders. Semin. Hematol. 3:351-375.

4. Cartwright, G.E., and Lee, G.R. 1971. The anaemia of chronic disorders. Br. J. Haematol. 21:147-152.

5. Means, R.T. 1995. Pathogenesis of the anemia of chronic disease: a cytokine-mediated anemia. Stem Cells. 13:32-37.

6. Ludwiczek, S., Aigner, E., Theurl, I., and Weiss, G. 2003. Cytokine-mediated regulation of iron transport in human monocytic cells. Blood. 101:4148-4154.

7. Weinstein, D.A., et al. 2002. Inappropriate expression of hepcidin is associated with iron refractory anemia: implications for the anemia of chronic disease. Blood. 100:3776-3781.

8. Nicolas, G., et al. 2002. The gene encoding the iron regulatory peptide hepcidin is regulated by anemia, hypoxia, and inflammation. J. Clin. Invest. 110:1037-1044. doi:10.1172/JCI200215686.

9. Nemeth, E., et al. 2003. Hepcidin, a putative mediator of anemia of inflammation, is a type II acutephase protein. Blood. 101:2461-2463.

10. Nemeth, E., et al. 2004. IL-6 mediates hypoferremia of inflammation by inducing the synthesis of the iron regulatory hormone hepcidin. J. Clin. Invest. 113:1271-1276. doi:10.1172/JCI200420945.

11. Pigeon, C., et al. 2001. A new mouse liver-specific gene, encoding a protein homologous to human antimicrobial peptide hepcidin, is overexpressed during iron overload. J. Biol. Chem. 276:7811-7819.

12. Muckenthaler, M., et al. 2003. Regulatory defects 
in liver and intestine implicate abnormal hepcidin and Cybrd 1 expression in mouse hemochromatosis. Nat. Genet. 34:102-107.

13. Adamsky, K., et al. 2004. Decreased hepcidin mRNA expression in thalassemic mice. Br.J. Haematol. 124:123-124.

14. Dallalio, G., Fleury, T., and Means, R.T. 2003.
Serum hepcidin in clinical specimens. Br. J. Haematol. 122:996-1000.

15. Roetto, A., et al. 2003. Mutant antimicrobial peptide hepcidin is associated with severe juvenile hemochromatosis. Nat. Genet. 33:21-22.

16. Nicolas, G., et al. 2001. Lack of hepcidin gene expression and severe tissue iron overload in upstream stimulatory factor 2 (USF2) knockout mice. Proc. Natl. Acad. Sci. U. S. A. 98:8780-8785.

17. Weinberg, E.D. 1986. Iron, infection and neoplasia. Clin. Physiol. Biochem. 4:50-60.

18. Park, C.H., Valore, E.V., Waring, A.J., and Ganz, T. 2001. Hepcidin, a urinary antimicrobial peptide synthesized in the liver. J. Biol. Chem. 276:7806-7810.

\title{
Hold the antioxidants and improve plasma lipids?
}

\author{
Ronald M. Krauss
}

Children's Hospital Oakland Research Institute, Oakland, California, USA.

\begin{abstract}
Intrahepatic proteolysis is a major determinant of secretion of ApoB-containing lipoproteins into plasma. Stimulation of post-ER presecretory proteolysis (PERPP) of ApoB by n-3 polyunsaturated fatty acids has been found to result in reduced secretion of VLDL particles by hepatocytes. A new study has shown that this stimulation is promoted by pro-oxidant conditions that result in increased hepatic lipid hydroperoxide content (see the related article beginning on page 1277). Conversely, PERPP is suppressed by antioxidants and by saturated fatty acids, which are not susceptible to lipid peroxidation. Hence reduction of oxidative stress may have the unexpected side effect of increasing plasma lipid levels.
\end{abstract}

Dietary fats with differing fatty acid composition can influence plasma lipid levels by modulating hepatic production and clearance of lipoproteins (1), as well as by altering activity of cholesteryl ester transfer protein (2). Suppression and stimulation of hepatic LDL receptor activity are major determinants, respectively, of the effects of saturated and polyunsaturated fatty acids on plasma LDL clearance (1), but the mechanisms for effects of specific fatty acids on hepatic lipoprotein production are less well understood. This is in large part due to the multiple influences of fatty acids on processes that regulate hepatic lipid production and storage, and processing of $\mathrm{ApoB}$ in conjunction with lipoprotein synthesis and secretion (3).

Fatty acids are critically involved in hepatic lipoprotein production pathways that help maintain hepatic cholesterol homeostasis and the ability to respond to energy and other metabolic needs. Triglycerides influence a critical early step in secretion of ApoB-containing lipoproteins, namely the cotranslational binding of lipids to ApoB in the ER mediated by micro-

Nonstandard abbreviations used: ER-associated degradation (ERAD); microsomal triglyceride transfer protein (MTP); post-ER presecretory proteolysis (PERPP); thiobarbituric acid-reactive substance (TBARS).

Conflict of interest: The author has declared that no conflict of interest exists.

Citation for this article: J. Clin. Invest. 113:1253-1255 (2004). doi:10.1172/JCI200421637. somal triglyceride transfer protein (MTP). The resulting protection of specific ApoB domains from proteolysis, termed ER-associated degradation (ERAD), leads either to secretion of a relatively lipid-depleted particle or to further, posttranslational lipidation (3) (Figure 1). The latter may occur in a graded manner in the ER, the vesicular tubular complex, and/or the Golgi apparatus, or by fusion with a preformed lipid droplet in the ER by a process that is not dependent on MTP.

\section{Post-ER presecretory proteolysis and} hepatic lipid hydroperoxide content

Recently, Fisher et al. have identified another degradative process that can modulate hepatic secretion of more mature ApoB-containing lipoproteins (4). They have found that inhibition of hepatic ApoB secretion by $n-3$ polyunsaturated fatty acids occurs via activation of this process, which has been designated postER presecretory proteolysis (PERPP) (4). In this issue of the JCI, Pan, Fisher, and colleagues have now shown, using several lines of evidence, that this effect is mediated by fatty acid peroxidation, and that it also occurs with n-6 polyunsaturated fatty acids (5). Moreover, the finding that ApoB degradation is stimulated by pro-oxidant conditions and inhibited by antioxidants raises the question of whether variation in intrahepatic oxidative stress contributes to physiologic and/or pathologic modulation of ApoB-containing lipoprotein metabolism. There is indeed abundant evidence that consumption of supplements of longer-chain n-3 marine-derived polyunsaturated fatty acids (eicosapentaenoic and docosahexanoic acids) can lower plasma triglyceride and VLDL levels in humans (6). Recently, it has been shown that population variation in dietary intake of a plantderived n-3 polyunsaturated fatty acid, linolenic acid, is significantly associated with plasma triglyceride levels, independent of other nutrients, including longerchain n-3 polyunsaturated fatty acids (7). As Pan et al. point out, however, in their study linolenic acid suppressed ApoB secretion from rat hepatoma cells to a greater extent than could be accounted for by the relation of secretion to intrahepatic lipid hydroperoxide content as assessed by levels of thiobarbituric acid-reactive substances (TBARSs) (5). It should be noted, however, that TBARSs do not represent the full spectrum of products of lipid peroxidation, such as F2-isoprostanes. Moreover, despite the capacity for peroxidation of both n-3 and n-6 polyunsaturated fatty acids, the latter have minimal and generally nonsignificant effects on plasma triglyceride levels in humans (8). Hence increased lipid peroxidation of n-3 fatty acids does not fully explain the effects of these fatty acids on hepatic ApoB-containing lipoprotein secretion. Other effects, such as reduced lipid synthesis, may also play a role.

\section{Oxidative stress and modulation of pathways for hepatic lipoprotein secretion}

The direct relation of the number of unsaturated bonds to lipid peroxidation is consistent with the lack of a suppressive effect of saturated fatty acids on plasma triglyceride levels. In contrast, a saturated fatty acid, myristic acid, was found to stimulate secretion of ApoB-containing 